УДК $519.21+62$

\author{
Y. M. Chabanyuk, A. V. Nikitin, U. T. Khimka \\ CONTROL PROBLEM FOR THE IMPULSE PROCESS \\ UNDER STOCHASTIC OPTIMIZATION PROCEDURE \\ AND LEVY CONDITIONS
}

\begin{abstract}
Y. M. Chabanyuk, A. V. Nikitin, U. T. Khimka. Control problem for the impulse process under stochastic optimization procedure and Levy conditions, Mat. Stud. 55 (2021), 107-112.

A stochastic approximation procedure and a limit generator of the original problem are constructed for a system of stochastic differential equations with Markov switching and impulse perturbation under Levy approximation conditions with control, which is determined by the condition for the extremum of the quality criterion function.

The control problem using the stochastic optimization procedure is a generalization of the control problem with the stochastic approximation procedure, which was studied in previous works of the authors. This generalization is not simple and requires non-trivial approaches to solving the problem.

In particular we discuss how the behavior of the boundary process depends on the prelimiting stochastic evolutionary system in the ergodic Markov environment. The main assumption is the condition for uniform ergodicity of the Markov switching process, that is, the existence of a stationary distribution for the switching process over large time intervals. This allows one to construct explicit algorithms for the analysis of the asymptotic behavior of a controlled process. An important property of the generator of the Markov switching process is that the space in which it is defined splits into the direct sum of its zero-subspace and a subspace of values, followed by the introduction of a projector that acts on the subspace of zeros.

For the first time, a model of the control problem for the diffusion transfer process using the stochastic optimization procedure for control problem is proposed. A singular expansion in the small parameter of the generator of the three-component Markov process is obtained, and the problem of a singular perturbation with the representation of the limiting generator of this process is solved.
\end{abstract}

Introduction. Analyzing the state of the art concerning asymptotic properties of stochastic evolution models reveals that a complete theory is still to be worked out. The models which are given by stochastic differential equations with Markov switchings and impulse or continuous-type perturbations in the classical schemes of averaging or diffusion approximation are well understood ([2]-[4]). Also, the asymptotic behavior was investigated of impulse processes with Markov switchings under the conditions of Levy approximation ([1], [8]-[11]). Thus, it seems natural to develop a theory of evolution equations with Markov switchings and random perturbations in nonclassical approximation schemes. Establishing convergence of the stochastic optimization procedure is an important puprose of system analysis in the

2010 Mathematics Subject Classification:60H30.

Keywords: random evolution; Levy approximation conditions; stochastic optimization procedure; Markov process.

doi:10.30970/ms.55.1.107-112

(C) Y. M. Chabanyuk, A. V. Nikitin, U. T. Khimka, 2021 
uncertainties, which can be modeled using an ergodic Markov environment. The relevance of determining new properties and generalizations of optimization algorithms that use randomness in the process of finding the optimum is evidenced by numerous applications in control theory, information transfer theory, and also in solving nonparametric problems of mathematical statistics. In the present paper, we focus on the study of the evolutionary system in the form of a perturbed controlled impulse process with Markov switching under the conditions of the existence of a single extremum point of the function for assessing the quality of control. We consider some prelimit evolution models with a small normalization parameter. We find the form of the limit generators for the impulse processes and the control of dynamical system in the schemes of the Levy approximation, and the stochastic optimization. Further, we provide conditions which ensure weak convergence of a controlled evolution model with Markov switching and impulse perturbation (assuming uniqueness of the equilibrium point for the quality criterion for which the stochastic optimization procedure is given). It is important that the asymptotic behavior of the limit process is concluded with the help of the analysis of parameters of the pre-limit system.

1. Problem formulation. We investigate the stochastic evolution system in the ergodic Markovian environment given by a stochastic evolutionary equation ([7], [8], [10])

$$
d y^{\varepsilon}(t)=C\left(y^{\varepsilon}(t), x\left(t / \varepsilon^{2}\right)\right)+d \eta^{\varepsilon}\left(t, u^{\varepsilon}(t)\right)
$$

where $y^{\varepsilon}(t)$ is a solution, $x(t), t \geq 0$, is a uniformly ergodic Markov process, that is defined on a standard phase space $(X, \mathbf{X})([3])$ by the generator

$$
\mathbf{Q} \varphi(x)=q(x) \int_{X} P(x, d y)[\varphi(x)-\varphi(y)]
$$

on the Banach space $B(X)$ of real valued bounded functions with the supremum norm $\|\varphi(x)\|=\sup _{x \in X}|\varphi(x)| ; u^{\varepsilon}(x)$ is a control function. A stochastic kernel $P(x, B), x \in X$, $B \in \mathbf{X}$ defines uniformly ergodic embedded Markov chain $x_{n}=x\left(\tau_{n}\right), n \geq 0$, that has stationary distribution $\rho(B), B \in \mathbf{X}$. Stationary distribution $\pi(B), B \in \mathbf{X}$ for Markov process $x(t), t \geq 0$, is defined by relation ([3])

$$
\pi(d x) q(x)=q \rho(x), \quad q=\int_{X} \pi(d x) q(x) .
$$

The impulse perturbation process $\eta^{\varepsilon}(t), t \geq 0$, is given by the relation

$$
\eta^{\varepsilon}\left(t, u^{\varepsilon}(t)\right)=\int_{0}^{t} \eta^{\varepsilon}\left(s, u^{\varepsilon}(s), x\left(s / \varepsilon^{2}\right)\right) d s
$$

where a set of processes with independent increments $\eta^{\varepsilon}(t, u, x), t \geq 0, x \in X, u \in \mathbb{R}$, is determined by the generators

$$
\Gamma^{\varepsilon}(x) \varphi(u, w)=\varepsilon^{-2} \int_{\mathbb{R}} \varphi(u, w+v)-\varphi(u, w) \Gamma^{\varepsilon}(d v, x),
$$

where $x \in X$ and the following Levy's approximation conditions are satisfied $([2,3])$ :

L1. The approximation of averages ([4])

$$
\begin{gathered}
\int_{\mathbb{R}} v \Gamma^{\varepsilon}(d v, x)=\varepsilon a_{1}(x)+\varepsilon^{2}\left(a_{2}(x)+\theta_{a}(x)\right), \theta_{a}(x) \rightarrow 0, \varepsilon \rightarrow 0, \\
\int_{\mathbb{R}} v^{2} \Gamma^{\varepsilon}(d v, x)=\varepsilon\left(b(x)+\theta_{b}(x)\right), \theta_{b}(x) \rightarrow 0, \varepsilon \rightarrow 0 .
\end{gathered}
$$


L2. The condition imposed on the distribution function

$$
\int_{\mathbb{R}} g(v) \Gamma^{\varepsilon}(d v, x)=\varepsilon^{2}\left(\Gamma_{g}(x)+\theta_{g}(x)\right), \theta_{g}(x) \rightarrow 0, \varepsilon \rightarrow 0,
$$

for all $g(v) \in C^{2}(\mathbb{R})$ (the space of real-valued bounded functions such that $g(v) /|v|^{2} \rightarrow 0$, $|v| \rightarrow 0)$, where measure $\Gamma_{g}(x)$ is bounded for all $g(v) \in C^{2}(\mathbb{R})$ and is defined by the relation (functions from the space $C^{2}(\mathbb{R})$ separate the measures):

$$
\Gamma_{g}(x)=\int_{\mathbb{R}} g(v) \Gamma_{0}(d v, x), g(v) \in C^{3}(\mathbb{R}) .
$$

L3. The uniform quadratic integrability: $\sup \lim _{c \rightarrow \infty} \int_{|v|>c} v^{2} \Gamma_{0}(d v, x)=0$.

As a toy example of a random variable that satisfies the conditions of the Levy approximation, we can cite the following $\alpha$

$$
P\{\alpha=b\}=\varepsilon^{2} p, \quad P\left\{\alpha=\varepsilon a_{1}+\varepsilon^{2} b_{1}\right\}=1-\varepsilon^{2} p .
$$

Then we have

$$
\mathbf{E} \alpha=\varepsilon a_{1}+\varepsilon^{2}\left(b p+b_{1}\right)+o\left(\varepsilon^{2}\right), \quad \mathbf{E} \alpha^{2}=\varepsilon^{2}\left(b^{2} p+a_{1}^{2}\right)+o\left(\varepsilon^{2}\right) .
$$

2. Stochastic optimization procedure for control function. We assume that control function $u(t)$ for equation (1) is defined by the quality criterion $G(x, y, u)$, that has unique extremum for each value of process $y$ and for each state $x$ of Markov process $x(t)$ on the interval $\left[\tau_{i}, \tau_{i+1}\right)$. That is $u(t)$ is the unique extremum point of $G(x, y(t), u)$ for fixed $x$ and $y(t)$, in particular $G(x, y(t), u(t))=\max _{u} G(x, y(t), u)$ or $G(x, y(t), u(t))=\min _{u} G(x, y(t), u)$ for $t \in\left[\tau_{i}, \tau_{i+1}\right)$.

We assume also that $G(\cdot, \cdot, u) \in C^{2}\left(\mathbb{R}^{d}\right)$. Then $u(t)$ is completely determined by the system

$$
\frac{\partial G(y(t), x(t), u(t))}{\partial u_{k}}=0 \quad(k=\overline{1, d}) .
$$

So we consider a stochastic optimization procedure $[9,12]$ for the control function $u(t)$

$$
d u^{\varepsilon}(t)=\alpha(t) \nabla_{\beta(t)} G\left(y^{\varepsilon}(t), x\left(t / \varepsilon^{2}\right), u^{\varepsilon}(t)\right) d t
$$

where

$$
\begin{gathered}
\nabla_{\beta(t)} G(\cdot, \cdot, u)=\left(G\left(\cdot, \cdot, u_{i}^{+}\right)-G\left(\cdot, \cdot, u_{i}^{-}\right)\right) / 2 \beta(t), i=\overline{1, d} \\
u_{i}^{ \pm}=u_{i} \beta(t) e_{i}, e_{i}=(0, \ldots, 1,0, \ldots, 0), i=\overline{1, d} .
\end{gathered}
$$

General initial conditions have a form

$$
y(0)=y_{0} ; x(0)=x_{0} ; u(0)=u_{0} .
$$

Functions $\alpha(t), \beta(t), t>0$ are satisfying the relations $\alpha(t) \rightarrow 0, \beta(t) \rightarrow 0(t \rightarrow \infty)$.

\section{Main results.}

Theorem 1. We assume the balance condition $\int_{X} \pi(d x) a_{1}(x)=0$ holds true, $C(y, x) \in$ $C\left(\mathbb{R}^{d}, \mathbf{X}, \alpha(t) \rightarrow 0, \beta(t) \rightarrow 0\right.$ as $t \rightarrow \infty$, and quality criterion $G(y, u, x) \in C\left(\mathbb{R}^{d}, \mathbf{X}, \mathbb{R}^{d}\right)$. Then weak convergence

$$
\left(y^{\varepsilon}(t), u^{\varepsilon}(t), \eta^{\varepsilon}(t)\right) \Rightarrow(\hat{y}(t), \hat{u}(t), \hat{\eta}(t)), \varepsilon \rightarrow 0
$$


holds true, where limit process $(\hat{y}(t), \hat{u}(t), \hat{\eta}(t))$ is defined by the generator

$$
\mathbf{M} \varphi(y, u, w)=\mathbf{L} \varphi(y, u, w)+\mathbf{B}_{t} \varphi(y, u, w)
$$

where

$$
\begin{gathered}
\mathbf{L} \varphi(y, u, w)=\mathbf{C}(y) \varphi(y, u, w)+\mathbf{\Gamma} \varphi(y, u, w), \mathbf{C}(y) \varphi(y)=\hat{C}(y) \varphi^{\prime}(y), \hat{C}(y)=\int_{X} \pi(d y) C(x, y), \\
\Gamma \varphi(u, w)=\hat{a}_{2} \varphi_{w}^{\prime}(u, w)+\frac{1}{2} \sigma^{2} \varphi_{w w}^{\prime \prime}(u, w)+\int_{R}[\varphi(u, w+v)-\varphi(u, w)] \hat{\Gamma}_{0}(d v), \\
\hat{a}_{2}=\int_{X} \pi(d x)\left(a_{2}(x)-a_{0}(x)\right), \sigma^{2}=\int_{X} \pi(d x)\left(b(x)-b_{0}(x)\right)+2 \int_{X} \pi(d x) a_{1}(x) R_{0} a_{1}(x), \\
a_{0}(x)=\int_{R} v \Gamma_{0}(d v, x), b_{0}(x)=\int_{R} v^{2} \Gamma_{0}(d v, x), \hat{\Gamma}_{0}(v)=\int_{X} \pi(d x) \Gamma_{0}(v, x), \\
\mathbf{B}_{t} \varphi(y, u, w)=\alpha(t) \nabla_{\beta(t)} G(y, u) \varphi_{u}^{\prime}(y, u, w), \nabla_{\beta(t)} G(y, u)=\int_{X} \nabla_{\beta(t)} G(y, u, x) \pi(d x) .
\end{gathered}
$$

Corollary 1. Limit process $(\hat{y}(t), \hat{u}(t))$ for control problem (5), (6), (7), is determined by stochastic differential equations

$$
d \hat{y}(t)=a(\hat{y}(t)) d t+d \eta((\hat{y}(t), \hat{u}(t))), \quad d \hat{u}(t)=\alpha(t) \nabla_{\beta(t)} G(\hat{y}(t), \hat{u}(t)) d t,
$$

under initial conditions (7).

Corollary 2. Assuming that an impulse perturbation process $y(t)$, defined as series scheme by the stochastic differential equation

$$
d y^{\varepsilon}(t)=C\left(y^{\varepsilon}(t), x\left(t / \varepsilon^{2}\right), u^{\varepsilon}(t)\right) d t+d \eta^{\varepsilon}\left(t, u^{\varepsilon}(t)\right),
$$

with control determined by stochastic optimization procedure (5) and

$$
\{C(y, x, u), G(y, x, u)\} \subset C^{2,0,2}\left(\mathbb{R}^{d}, X, \mathbb{R}^{d}\right) .
$$

Then weak convergence $\left(y^{\varepsilon}(t), u^{\varepsilon}(t)\right) \Rightarrow(\hat{y}(t), \hat{u}(t)), \varepsilon \rightarrow 0$, holds true, where $\varepsilon$ is small enough, and the limit process $(\hat{y}(t), \hat{u}(t))$ is determined by generator

$$
\mathbf{A}(y, u) \varphi(y, u)=a(y, u) \varphi_{y}^{\prime}(y, u)+\alpha(t) \nabla_{\beta(t)} G(y, u) \varphi_{u}^{\prime}(y, u), \quad a(y, u)=\int_{X} \pi(d x) a(y, u, x),
$$

on test functions $\varphi(y, u) \in C^{3,2}\left(\mathbb{R}^{d}, \mathbb{R}^{d}\right)$.

Lemma 1. Generator $\mathbf{L}^{\varepsilon}(x)$ of problem (1), (5) under conditions of Theorem 1 has the asymptotical representation

$$
\begin{gathered}
\mathbf{L}^{\varepsilon}(x) \varphi(y, u, w, x)=\varepsilon^{-2} \mathbf{Q} \varphi(y, u, w, x)+\varepsilon^{-1} \boldsymbol{\Gamma}_{1}(x) \varphi(y, u, w, x)+ \\
\quad+\mathbf{C}(y) \varphi(y, u, w, x)+\mathbf{B}_{t}(x) \varphi(y, u, w, x)+\hat{\theta}_{w}^{\varepsilon}(x) \varphi(y, u, w, x),
\end{gathered}
$$

where $\mathbf{B}_{t}(x) \varphi(y, u, w, x)=\alpha(t) \nabla_{\beta(t)} G(y, u, x) \varphi_{u}^{\prime}(y, u, w, x)$, and the remainder term $\hat{\theta}_{w}^{\varepsilon}$ satisfies condition $\left\|\hat{\theta}_{w}^{\varepsilon}(x) \varphi(y, u, w, x)\right\| \rightarrow 0$ when $\varepsilon \rightarrow 0$.

The proof of Lemma 1 is performed according to the scheme as in [5].

We obtain the result of Theorem 1 as the solution of singular perturbation problem [6] for generator $\mathbf{L}^{\varepsilon}(x)$ at test functions [7]

$$
\varphi^{\varepsilon}(y, u, w, x)=\varphi(y, u, w)+\varepsilon \varphi_{1}(y, u, w, x)+\varepsilon^{2} \varphi_{2}(y, u, w, x)
$$

under conditions of Theorem 1. 
Theorem 2. We assume that the Lyapunov function $V(y, u)$ of averaged system exists and it has a form $\frac{d u}{d t}=G^{*}(y, u)$, where $G^{*}(\cdot, u)=\operatorname{grad} G(\cdot, u)=\left\{\frac{\partial G}{\partial u_{1}}, \ldots, \frac{\partial G}{\partial u_{d}}\right\}$ for arbitrary value of the process $y$ and averaged quality function satisfies global Lipschitz condition

Y1: $G^{*}(y, u) V^{\prime}(y, u) \leq-c_{0} V(y, u)$;

$Y 2$ : for $\beta(t)>0\left|\nabla_{\beta(t)} G(y, u)-G^{*}(y, u)\right| \leq c_{2} \beta(t), c_{2}>0$;

Y3: $\left|\nabla_{\beta(t)} G(y, x, u) R_{0}\left[\nabla_{\beta(t)} \tilde{G}(y, x, u) V^{\prime}(y, u)\right]_{u}^{\prime}\right| \leq c_{1}(1+V(y, u))$.

We assume further

F1: $\int_{t_{0}}^{\infty} \alpha(t) d t=\infty, \quad \int_{t_{0}}^{\infty} \alpha(t) \beta(t) d t<\infty$.

Then for each $\varepsilon$ small enough, for solution the control problem (5), (6) and for arbitrary value of process $y$ relation

$$
\mathbb{P}\left\{\lim _{\varepsilon \rightarrow 0} u^{\varepsilon}(t)=u(y)\right\}=1
$$

holds true.

Lemma 2. We have an asymptotical representation for a generator of control $\mathbf{B}_{t}(x)$

$$
\mathbf{B}_{t}(x) V^{\varepsilon}(y, u, w, x)=\mathbf{B}_{t} V(y, u)+\varepsilon \theta_{u}(x) V(y, u),
$$

where $\left\|\theta_{u}(x) V(y, u)\right\| \leq M, M>0$, and

$$
V^{\varepsilon}(y, u, w, x)=V(y, u)+\varepsilon V_{1}(y, u, w, x)+\varepsilon^{2} V_{2}(y, u, w, x)
$$

is the perturbed Lyapunov function.

Proof of Theorem 2. From Lemma 2 and conditions Y1, Y2 and Y3 we obtain estimation

$$
\mathbf{B}_{t}(x) V^{\varepsilon}(y, u, w, x) \leq-c_{0} \alpha(t) V(y, u)+c^{*} \alpha(t) \beta(t)(1+V(y, u)) .
$$

Then from condition F1 and Nevelson-Khasminskiy Theorem [9] we have Theorem 2.

Remark 1. Weak convergence of process $\left(y^{\varepsilon}(t), u^{\varepsilon}(t), \eta^{\varepsilon}(t)\right) \Rightarrow(\hat{y}(t), \hat{u}(t), \hat{\eta}(t)), \varepsilon \rightarrow 0$, follows from convergence of the corresponding generators under condition of compactness of pre-limited set of processes $\left.\left(y^{\varepsilon}(t), u^{\varepsilon}(t)\right)\right)$. In particular, the corresponding theorems on the compactness of processes with independent increments in Levy approximation scheme have been proved in [4].

Remark 2. The stochastic optimization procedure for control $u$ gives optimal value $y_{u}$ for any impulse perturbation process $y$.

4. Conclusion. Weak convergence of stochastic processes is usually proved by checking the two conditions: (a) tightness of the distributions of the converging processes which ensures the existence of a converging subsequence and (b) uniqueness of the weak limit. The passage to the limit can be done on the semigroups which correspond to the converging processes as well as on appropriate generators. 


\section{REFERENCES}

1. J. Jacod, A.N. Shiryaev, Limit theorems for stochastic processes. - Berlin: Springer-Verlag, 2003. - 601p.

2. V.S. Korolyuk, V.V. Korolyuk, Stochastic models of systems. - Dordrecht: Kluwer, 1999. - 185p.

3. V.S. Korolyuk, N. Limnios, Stochastic systems in merging phase space. - World Scientific, 2005. - 330p.

4. V.S. Korolyuk, N. Limnios, I.V. Samoilenko, Levy and Poisson approximations of switched stochastic systems by a semimartingale approach// Comptes Rendus Mathematique. - 2016. - V.354. - P. 723-728.

5. Y.M. Chabanyuk, A.V. Nikitin, U.T. Khimka, Asymptotic properties of the impulse perturbation process with control function under Levy approximation conditions// Mat. Stud. - 2019. - V.52, №1. - P. 96-104.

6. A.V. Nikitin, U.T. Khimka, Asymptotics of normalized control with Markov switchings// Ukr. Math. J. - 2017. - V.68, №8. - P. 1252-1262.

7. I.V. Samoilenko, A.V. Nikitin, Differential equations with small stochastic terms under the Levy approximating conditions // Ukr. Math. J. - 2018. - V.69, №9. - P. 1445-1454.

8. A.V. Nikitin, Asymptotic properties of a stochastic diffusion transfer process with an equilibrium point of a quality criterion// Cybern. Syst. Anal. - 2015. - V.51. - P. 650-656.

9. M.B. Nevelson, R.Z. Khas'minskii, Stochastic approximation and recursive estimation. - Moscow: Nauka, 1972. - 298p.

10. A.V. Nikitin, Asymptotic dissipativity of stochastic processes with impulsive perturbation in the Levy approximation scheme// Journal of Automation and Information Sciences. - 2018. - V.50, №4. - P. 205211.

11. G. Papanicolaou, D. Stroock, S.R.S. Varadhan, Martingale approach to some limit theorems// Duke turbulence conference (Durham, NC, April 23-25, 1976), Duke University Mathematics Series III, New York: Duke University, 1977. - 120 p.

12. U.T. Khimka, Ya.M. Chabanyuk, A difference stochastic optimization procedure with impulse perturbation // Cybern. Syst. Anal. - 2013. - V.49. - P. 768-773.

Politechnika Lubelska, Lublin, Poland

Ivan Franko National University of Lviv

Lviv, Ukraine

yaroslav.chabanyuk@lnu.edu.ua

National University of Life and Environmental Sciences of Ukraine

Kyiv, Ukraine

nikitin2505@nubip.edu.ua

Ivan Franko National University of Lviv

Lviv, Ukraine

ulyana.khimka@lnu.edu.ua

Received 22.08.2020

Revised 15.10.2020 